\title{
Ureaplasma urealyticum as a cause of pneumonia in preterm infants: analysis of the white cell response
}

\author{
A Panero, L Pacifico, N Rossi, M Roggini, C Chiesa
}

\begin{abstract}
The tracheal isolation of Ureaplasma urealyticum from critically ill infants was investigated to determine if the organism was associated with an inflammatory response. Twenty nine neonates consecutively admitted for acute respiratory disease, with birthweights of $<1301 \mathrm{~g}$ and no evidence of viral, chlamydial, or bacterial infections, were identified. Culture results for ureaplasmas were correlated with white cell counts and clinical and radiographic features. Sixteen infants had tracheal aspirates and/or blood specimens positive for $U$ urealyticum. Pneumonia was diagnosed more frequently in the $U$ urealyticum positive infants than in the 13 who were negative for the organism. The mean total white cell count, absolute neutrophil, and band form counts were significantly higher in the $U$ urealyticum positive group than in the negative group.

These data suggest that $U$ urealyticum can induce an inflammatory response in selected individuals who present with clinical, radiographic, and, in some instances, histological features of pneumonia.
\end{abstract}

(Arch Dis Child 1995; 73: F37-F40)

Keywords: Ureaplasma pneumonia, white cell counts, preterm infants.

During the past decade there has been increased interest in Ureaplasma urealyticum and its possible role in perinatal morbidity and mortality, including sepsis, meningitis, and pneumonia. ${ }^{1-4}$ Therefore, given the pathogenic potential of this organism, the ability to target this population for a marker of severity of inflammation in order to direct treatment against $U$ urealyticum would be welcome.

$U$ urealyticum colonisation of the nasopharynx and/or trachea of preterm neonates in the absence of other microbial pathogens is associated with an increased white cell count, indicative of a true inflammatory process. ${ }^{5}$ During the course of an ongoing prospective study on the incidence and outcome of $U$ urealyticum infection in neonates, we discovered three neonates with $U$ urealyticum infection of the respiratory tract who had a substantial increase in white cell count. ${ }^{6}$ These observations needed to be confirmed and extended, and it was with this aim that we reviewed all neonates with acute respiratory distress recently admitted to the neonatal intensive care unit who had had specimens cultured for mycoplasmas, and we correlated culture results with white cell counts, clinical and chest radiograph findings, paying particular attention to pneumonic changes.

\section{Methods}

All infants with acute respiratory distress admitted consecutively to the neonatal intensive care unit at the University of Rome, Italy, from October 1993 to September 1994 $(n=55)$ were included in the study. Data on maternal and perinatal risk factors (rupture of membranes, maternal fever, perinatal asphyxia and Apgar scores) were obtained by chart abstraction. The gestational age of all infants was determined using the Dubowitz score.

Samples were obtained for cultures of $U$ urealyticum within the first day of life, during the third day of life, and subsequently in some cases if the patient's respiratory condition deteriorated. Tracheal aspirates were collected by mechanical suction through a catheter passed into the distal trachea through an endotracheal tube and were immediately placed into $2 \mathrm{ml}$ of urea containing mycoplasmal broths. $^{7}$ Blood was collected for culture of mycoplasmas either by peripheral venepuncture or from an umbilical catheter and inoculated into mycoplasmal broths. Subcultures for identification of $U$ urealyticum were done on differential agar medium A7. ${ }^{8}$ Broths were incubated at $37^{\circ} \mathrm{C}$ under atmospheric conditions, and agar plates under $5 \% \mathrm{CO}_{2}$. All cultures were held for seven days. $U$ urealyticum was identified by its characteristic morphological colonies and urease production.

Tracheal and blood specimens were cultured for aerobic and anaerobic organisms. Nasopharyngeal and tracheal specimens were also cultured for Chlamydia trachomatis and viruses.

White cell counts were performed by automated methods and were corrected for the presence of increased nucleated red blood cells. Differential counts were determined manually by duty personnel in accordance with standard methods.

\section{RADIOGRAPHIC ANALYSIS}

Chest radiographs were retrieved from the archives and reviewed by a paediatric radiologist (MR) who had no knowledge of the clinical history. There was agreement between the first and second interpretations. 
Characteristics of infants studied with and without $\mathrm{U}$ urealyticum infection

\begin{tabular}{llll}
\hline & \multicolumn{2}{l}{ U urealyticum infection } & \\
\cline { 2 - 3 } & Present $(n=16)$ & Absent $(n=13)$ & P value \\
\hline Birthweight (g) & $963(150)$ & $978(201)$ & NS \\
Gestational age (week) & $27(1 \cdot 4)$ & $29(1 \cdot 4)$ & $0 \cdot 001$ \\
Gender M:F & $7: 9$ & $3: 10$ & $\mathrm{NS}$ \\
Vaginal delivery & 10 & 2 & $0 \cdot 047$ \\
Birth asphyxia & 12 & 6 & $\mathrm{NS}$ \\
Exogenous surfactant & 9 & 6 & $\mathrm{NS}$ \\
Evidence of pneumonia† & 14 & $7(9)$ & $0 \cdot 0017$ \\
Total days of assisted ventilation & $16(10)$ & $12(20)$ & $0 \cdot 033$ \\
Total days of oxygen therapy & $43(40)$ & 1 & $0 \cdot 026$ \\
Intraventricular haemorrhage & 5 & 3 & $\mathrm{NS}$ \\
Patent ductus arterious & 3 & 1 & $\mathrm{NS}$ \\
Death & 4 & $\mathrm{NS}$ \\
\hline
\end{tabular}

${ }^{\star}$ Mean (SD). †Radiographic and/or postmortem. NS=not significant.

Pneumonia was defined as the presence of one or more of the following: (1) radiating peripheral streakiness; (2) coarse patchy parenchymal infiltrates or small diffuse nodules; (3) subtle, hazy, or nodular basilar infiltrates; or (4) diffuse granularity indistinguishable from hyaline membrane disease, but with better than expected aeration. ${ }^{910}$

\section{STATISTICS}

The means of the numerical data were tested for significant differences using the two tailed independent $t$ test. Frequency incidences were analysed using the $\chi^{2}$ test incorporating Yates' correction, where indicated. The level of significance was set at $0 \cdot 05$.

\section{Results}

Specimens were obtained for the culture of $U$ urealyticum from the lower respiratory tract as well as from the blood of 55 infants.

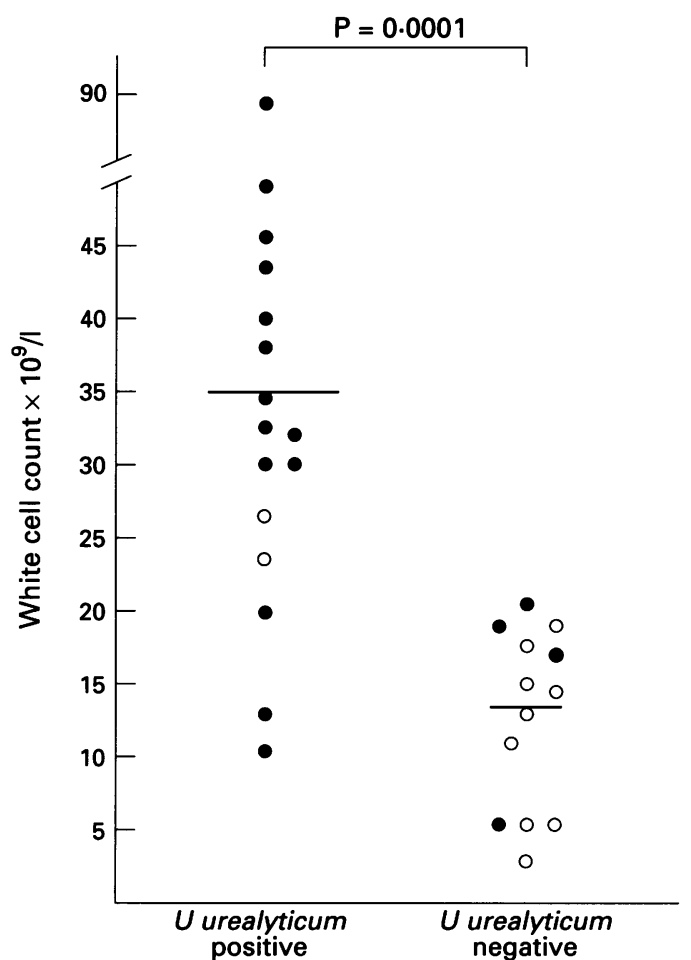

Figure 1 Total white cell count on day 3 of life in 16 neonates positive for $\mathrm{U}$ urealyticum compared with 13 neonates negative for $\mathrm{U}$ urealyticum. Solid line represents mean values: $=$ with evidence of pneumonia; $O=$ withou evidence of pneumonia.
Neonates with birthweights of $>1300 \mathrm{~g}$ $(n=17)$, systemic bacterial infections $(n=3)$, a tracheal aspirate positive for other potential respiratory pathogens, such as Streptococcus agalactiae, Escherichia coli, Staphylococcus aureus, and Chlamydia trachomatis $(n=4)$, or serious congenital malformations $(n=2)$ were excluded for further analysis.

Among the 29 neonates eligible for this study, 16 were positive and 13 negative for $U$ urealyticum. Of the 16 positive newborns, 15 grew $U$ urealyticum from tracheal aspirates and one from blood as well as tracheal samples. Ten of these patients showed $U$ urealyticum growth in the aspirate specimens taken within the first 24 hours. The specimens of the remaining six patients became positive later; in five patients the second specimen (at the age of 48-72 hours) was the first positive one, and in one, the third (at the age of 5 days). Ureaplasmas were always isolated in quantities exceeding $10^{4}$ colony forming units $/ \mathrm{ml}$.

Blood and urine cultures for bacteria, and tracheal aspirate cultures for bacteria, chlamydiae and viruses were negative in all 29 neonates, as were serological studies for toxoplasmosis, rubella, cytomegalovirus infection, and herpes simplex virus infection.

The characteristics of the study patients are shown in the table. Infants with $U$ urealyticum were of significantly lower gestation. There were no differences between $U$ urealyticum positive and negative newborns with respect to gender, birth weight, incidence of maternal fever, as well as prolonged rupture of membranes and maternal hypertension. The isolation rate was significantly higher in neonates born by vaginal delivery. All the infants in both groups presented with acute respiratory disease requiring assisted ventilation and/or supplemental oxygen. Exogenous surfactant was used in nine of the 16 infants with, and in six of the 13 infants without, $U$ urealyticum. The incidence of patent ductus arterious and intraventricular haemorrhage was similar in the two groups.

A roentgenographic diagnosis of pneumonia was established in 13 of the $16 U$ urealyticum positive infants and in four of the 13 $U$ urealyticum negative infants $(81 \%$ v $31 \%$; $\mathrm{P}=0.006$ ).

Compared with infants whose ureaplasmal cultures were negative, those with $U$ urealyticum had a longer duration of respiratory symptoms, ventilatory support, and oxygen supplementation. Death occurred in four of the 16 Ureaplasma positive infants. The first two infants died on postnatal days 4 and 7 , respectively. In the first infant pneumonia was seen only at necropsy, while in the second one this was when the roentgenographic diagnosis of pneumonia was confirmed. Postmortem examination of the lung tissues showed the presence of patchy bilateral pneumonitis with polymorphonuclear cell infiltrates and areas of alveolar destruction, and yielded $U$ urealyticum in pure culture. The third infant who soon after birth had a chest $x$ ray picture indicative of pneumonia died at 24 hours of life because 


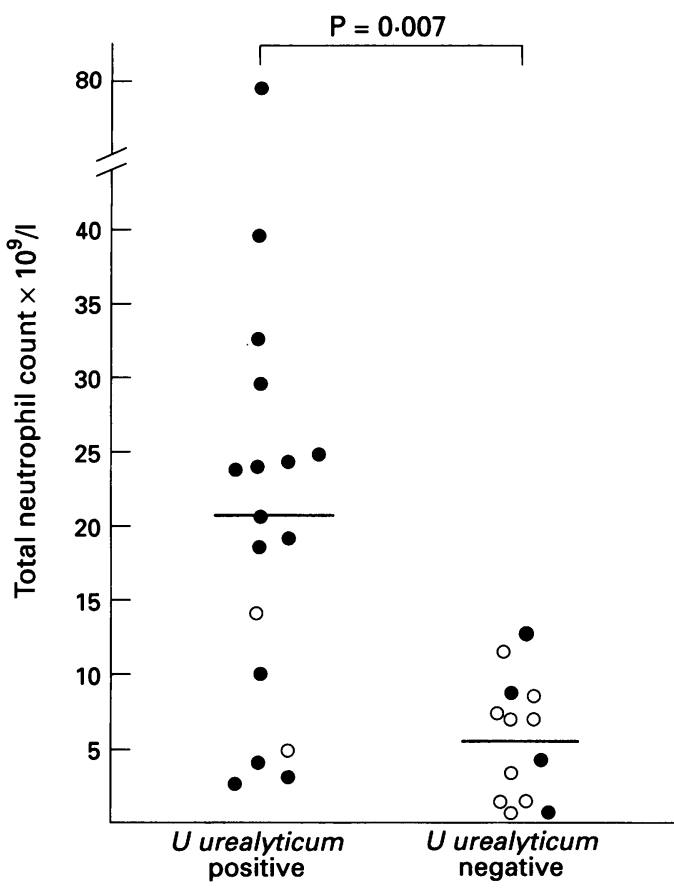

Figure 2 Total absolute neutrophil (polymorphonuclear plus band) count on day 3 of life in 16 neonates with $\mathrm{U}$ urealyticum and 13 neonates without. Solid line represents mean values: $=$ with evidence of pneumonia; $\mathrm{O}=$ without evidence of pneumonia.

of respiratory failure and intraventricular haemorrhage. Necropsy was not performed. The fourth infant, whose chest radiograph taken at 3 days of life showed bilateral pneumonitis, died of respiratory failure on day 28. A postmortem examination of the lungs showed extensive bilateral consolidation, multiple haemorrhages, moderate acute and chronic inflammation, necrosis of the bronchial epithelium, and interstitial fibrosis. Postmortem cultures were not performed. Among the 13 Ureaplasma negative infants, one died at 7 days of age because of hypertensive pneumothorax.

On day 3 of life, significant increases in the counts of total white cells and total absolute neutrophils (polymorphonoclear cells plus band forms) were observed in $U$ urealyticum positive infants compared with those who were negative (mean (SD) $35.2(18.6) \times 10^{9} / 1$ and $21.5(18.6) \times 10^{9} / 1 v 13.5(5.5) \times 10^{9} / 1$ and 6.2 (3.9) $\times 10^{9} / 1 ; \mathrm{P}=0.0001$ and $\mathrm{P}=0.007$, respectively) (figs 1 and 2). The band form counts were also significantly higher in the positive than in the negative group (mean (SD) 4.4 $(5.2) \times 10^{9} / 1$ v $\left.1.2(0.9) \times 10^{9} / 1 ; P=0.009\right)$. In the neonates positive for $U$ urealyticum, repeated white cell counts remained invariably raised until day 7 of life but gradually decreased.

When analysing the leucocyte response in accordance with pneumonic changes, the 14 $U$ urealyticum positive infants with roentgenographic $(n=13)$ and/or postmortem $(n=2)$ findings of pneumonia had a mean white cell count of $36.2(18.2) \times 10^{9} / 1$ while the four $U$ urealyticum negative infants with a roentgenographic diagnosis of pneumonia had a mean white cell count of $13.5(5.9) \times 10^{9} / 1$ $(P=0.004)$.

\section{Discussion}

The cause of lower respiratory disease in newborn babies is a diagnostic dilemma because pneumonia in the early neonatal period cannot be distinguished readily either clinically or radiologically from surfactant deficiency distress syndrome. In most nurseries it is therefore common practice to consider the possibility of sepsis or pneumonia in all clinically ill newborns with respiratory distress and to treat them with conventional antibiotics.

Although some investigators have reported apparently contradictory results in terms of the importance of $U$ urealyticum as a pathogen in newborns, recent prospective studies confirm the validity of case reports. These findings contend that under certain conditions $U$ urealyticum is a true pathogen capable of causing respiratory disease in newborn infants. ${ }^{41112}$ Additional evidence of the pathogenic potential of ureaplasmas in the respiratory tract comes from animal studies. Newborn pathogen-free mice inoculated intranasally with ureaplasmal strains isolated from the lungs of newborn human infants developed an acute, self-limiting, interstitial pneumonia. The lesions were most severe three days after inoculation and increased in severity by exposure to oxygen. ${ }^{13} 14$

That the $U$ urealyticum isolated from the tracheal aspirates of our patients represented true infection is suggested by the following reasons: (1) the organism was always isolated in pure culture and in high numbers; (2) $U$ urealyticum was the only organism isolated from the lung tissue of two infants with histological evidence of pneumonitis at postmortem examination; (3) compared with $U$ urealyticum negative infants, those who were positive had a significantly higher percentage of radiographic changes of pneumonia; (4) an inflammatory response, as shown by an increase in the mean total white cell count with both polymorphonuclear leucocytes and immature cells increasing in number, was observed in the $U$ urealyticum positive group.

Previous studies have shown that the leucocyte count is a sensitive screening test for neonatal infection. ${ }^{15} 16$ However, most studies were performed before $U$ urealyticum was implicated as a neonatal pathogen. Recently, Ohlsson et al observed an association between nasopharyngeal or endotracheal colonisation with $U$ urealyticum and raised white cell counts, but they did not interpret the leucocyte counts in conjunction with the clinical picture. ${ }^{5}$ Our results confirm and expand on the findings of the above report. In fact, in our study, the increase in white cell counts was a reliable indicator of the ability of the organism to induce an inflammatory response in selected individuals who presented with clinical, radiographic, and, in some instances, histological findings of pneumonia.

Routine screening for $U$ urealyticum is not warranted because many infants with ureaplasmas are asymptomatic and the organism often disappears without treatment. ${ }^{17}$ However, clinical and radiographic evidence of pneumonia, particularly in a preterm neonate, 
is an adequate justification for obtaining the appropriate specimens for mycoplasmal cultures. It is even more important to consider ureaplasmal infection when an ill neonate exhibits a leucocyte response and no other verifiable microbiological aetiologies of pneumonitis have been identified. Such neonates should be considered for antimicrobial treatment. Despite controversy stemming from non-standardised susceptibility testing, erythromycin is the drug of choice for treating neonatal ureaplasma infections not involving the central nervous system. The use of erythromycin is supported by its activity in vitro, limited data from clinical experience, and preliminary pharmacokinetic and safety studies. ${ }^{18}$

This study was in part supported by the Italian National Research Council (Grant No 94. 04983 PF41).

1 Quinn PA, Gillan JE, Markestad T, Iohn MA, Daneman A, $\mathrm{Lie} \mathrm{KI}$, et al. Intrauterine infection with Ureaplasma urealyticum as a cause of fatal neonatal pneumonia. Pediatr Infect Dis $₹$ 1985; 4: 538-43.

2 Brus F, van Waarde WM, Schoots C, Otomo SB. Fatal ureaplasmal pneumonia and sepsis in a newborn infant. Eur $\mathcal{F}$ Pediatr 1991; 150: 782-3.

3 Garland SM, Murton LJ. Neonatal meningitis caused by Ureaplasma urealyticum. Pediatr Infect Dis $\mathcal{f} 1987$; 6: 868-70.

4 Cassell GH, Waites KB, Crouse DT, Rudd PT, Canupp $\mathrm{KL}$, Stagno G, et al. Association of Ureaplasma ureKL, Stagno G, et al. Association of Ureaplasma urealyticum infection of the lower respiratory tract with
chronic lung disease and death in very-low-birth-weight infants. Lancet 1988; ii: $240-5$.

5 Ohlsson A, Wang E, Vearncombe M. Leukocyte counts and colonization with Ureaplasma urealyticum in preterm neonates. Clin Infect Dis 1993; 17: S144-7.

6 Panero A, Pacifico L, Rossi N, Bucci G, Chiesa C. Elevated white blood cell counts associated with Ureaplasma urealyticum colonization in preterm neonates. Clin Infect Dis 1994; 19: 980-1.

7 Shepard MC. Culture media for ureaplasmas. In: Razin S, Tully JG, eds. Methods in mycoplasmology, Vol 1. New Tully JG, eds. Methods in mycoplasm

8 Shepard MC, Lunceford CD. Differential agar medium (A7) for identification of Ureaplasma urealyticum (human T-mycoplasmas) in primary cultures of clinical material. f Clin Microbiol 1976; 3: 613-25.

9 Tudor J, Young L, Wigglesworth JS, Steiner RE. The value of radiology in the idiopathic respiratory distress syndrome: a radiological and pathological correlation study. Clin Radiol 1976; 27: 65-75.

10 Crouse DT, Odrezin GT, Cutter GR, Reese JM, Harwick WB, Waites KB, et al. Radiographic changes associated with tracheal isolation of Ureaplasma urealyticum from neonates. Clin Infect Dis 1993; 17: S122-30.

11 Rudd PT, Carrington D. A prospective study of chlamydial, mycoplasmal, and viral infections in a neonatal intensive mycoplasmal, and viral infections in a neo

12 Ollikainen J, Hiekkaniemi H, Korppi M, Sarkkinen H, Heinonen $K$. Ureaplasma urealyticum infection associated with acute respiratory insufficiency and

13 Rudd PT, Cassell GH, Waites KB, Davis JK, Duffy LB. Ureaplasma urealyticum pneumonia: experimental production and demonstration of age-related susceptibility. Infect Immun 1989; 57: 918-25.

14 Crouse DT, Cassell GH, Waites KB, Foster JM, Cassady G. Hyperoxia potentiates Ureaplasma urealyticum pneumonia in newborn mice. Infect Immun 1990; 58: 3487-93.

15 Xanthou $M$. Leucocyte blood picture in ill newborn babies. Arch Dis Child 1972; 47: 741-6.

16 Manroe BL, Rosenfeld CR, Browne R, Weinberg AG. The differential leucocyte count in the assessment and outcome of early-onset neonatal group B streptococcal disease. F Pediatr 1977; 91: 632-7.

17 Cassell GH, Waites KB, Crouse DT. Perinatal mycoplasmal infections. Clin Perinatol 1991; 18: 241-62.

18 Waites KB, Crouse DT, Cassell GH. Therapeutic considerations for Ureaplasma urealyticum infections in neonates. Clin Infect Dis 1993; 17: S208-14. 